\title{
Energy harvesting of cantilever beam system with linear and nonlinear piezoelectric model
}

\author{
Marek Borowiec ${ }^{\mathrm{a}}$ \\ Lublin University of Technology, Nadbystrzycka 36, 20-618 Lublin, Poland
}

Received 19 August 2015 / Received in final form 9 September 2015 Published online 20 November 2015

\begin{abstract}
The nonlinear beam with vertical combined excitations is proposed as an energy harvester. The nonlinearities are included both, in the beam model and also in the electrical subsystem. The system is modelled as a cantilever beam with included a tip mass and piezoelectric patches which convert the bending strains induced by both, the harmonic and the additive stochastic forces. The excitation affects in vertical directions by kinematic forcing into electrical charge. The first main goal is to analyse the dynamics of the electro-mechanical beam system and the influence of the mixed excitation forces into an effectiveness of the energy harvesting. Overcoming the potential barrier by the beam system is also analysed, where large output amplitudes occur. Such region of the vibration affects more power generation, which is crucial in terms of load resistors sensitivities. By increasing the additive noise level with fixed harmonic force it is observed the transition from single well oscillations to inter-well stochastic jumps. The second mail goal is analysing the influence of the piezoelectric nonlinear characteristic and compare the results to the linear piezoelectric cases. The output power is measured during different system behaviours provided by different piezoelectric characteristic as well as introduced stochastic components by modulated tip mass of the system.
\end{abstract}

\section{Introduction}

Nowadays self-powered devices are more and more common. The applications of small actuators and sensors work in small networks and stimulate new research ideas on ambient energy harvesting [2-5]. The solutions of frequency broadband nonlinear vibration have been developed in competition with photo-voltaic and thermal gradient approaches $[7,8]$. The effectiveness of frequency broadband devices is continuously growing [6]. In case of stable narrow band excitations, the approach is to introduce the mechanical system to resonate. Unfortunately it performs a weakness in transient and changeable conditions of ambient excitation [2,5]. Availabilities of energy harvesting at small levels, apart from resonance area is usually insufficient and requires a tuning process. Thus the expecting devices are broadband harvesters with nonlinear characteristics which are sufficient resistant to a specific amplitude or frequency

\footnotetext{
${ }^{a}$ e-mail: m.borowiec@pollub.pl
} 


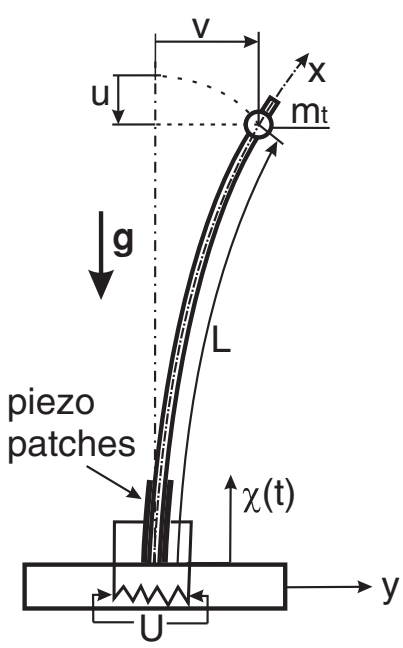

Fig. 1. The model of inverted beam harvester. The plane position of tip mass $m_{t}$ is given by $v$ and $u$ coordinates. The excitation in vertical direction has been provided by function $\chi(t)$. The vector $\mathbf{g}$ represents the gravitational acceleration.

excitation $[7,8]$. Thus a nonlinear mechanical resonators with a double well potentials looks to be promising [10-12]. In the paper of Friswell et al. [13] there is presented a piezoelastic system consisting of a cantilever beam with a tip mass. The mechanical system was mounted vertically and harmonically exciting in the horizontal direction at its base. In the experiment the two behaviours obtained. For a modified weight of the tip mass one can introduce a vertical position destabilization of the beam by buckling. The three different tip masses was simulated and also validated in experiment of the deterministic model. The results revealed cases of a linear system, a low natural frequency as well as both a non-buckled and a buckled beam. One can proved that the most practical configuration is a pre buckled case, where the system reaches a low natural frequency, and also a high level of harvested power. In the next paper [14], both the effects of random and the combined harmonic and stochastic excitation components in the same configuration of the system were studied. Similar approaches of piezoelastic devices have been also discussed in [15-22]. The aforementioned model $[13,14]$ is followed in the paper [1], where the analysis of this system is completed by simulating cases of harmonic and combined harmonic-stochastic excitations in vertical direction. In the present paper, results provided in [1] are recalculated for the sake of output averaged power and compared with different approach of piezoelectric subsystem modelling. The system considered in this paper includes also the bending effect due to the gravity force, in contrast to the magnetic systems discussed in previous papers $[12,17-21]$.

\section{The system modelling by equation of motion}

The model of the beam is presented in Fig. 1. An additional point mass $m_{t}$ had been included and the system has undergone a flexible body deflection due to base excitation defined by function $\chi(t)$. The system was exciting in vertically direction and function $\chi(t)$ had harmonically form with included noise component. A specified element of the beam mass $d m$ at $x$ position was moving in a vertical $\left(\chi-u_{x}\right)$ and in a horizontal $\left(v_{x}\right)$ directions. A rotation $\psi$ of the tip beam point $\left(m_{t}\right)$ has been 
also considered in the calculations. The rotation of the beam mass element $\psi_{x}$ was neglected because of the beams ratio dimensions. Due to the thickness of the beam was small compared with the length, the effect of shear deformation and rotary inertia of the beam were no significant.

The Eq. of motion has been derived from Largange method. The energies of the system, kinetic $T$ and potential $\Pi$ are as follow [13]:

$$
\begin{gathered}
T=\frac{1}{2} \rho A \int_{0}^{L}\left[\dot{v}_{x}(x, t)^{2}+\left(\dot{\chi}(t)-\dot{u}_{x}(x, t)\right)^{2}\right] \mathrm{d} x \\
+\frac{1}{2} m_{t}\left[\dot{v}(t)^{2}+(\dot{\chi}(t)-\dot{u}(t))^{2}\right]+\frac{1}{2} I_{0} \dot{\varphi}^{2} \\
\Pi=\frac{1}{2} E I \int_{0}^{L} \kappa(x, t)^{2} d x+g \rho A \int_{0}^{L}\left(-u_{x}(x, t)\right) d x+m_{t} g(-u(t)) .
\end{gathered}
$$

The potential energy consists of the elastic beam part and the gravity term of both, the beam and the tip masses. The beam elastic displacement gets relations:

$$
\cos \phi_{x}=\frac{d x-d u}{d x}=1-u_{x}^{\prime} \quad \text { and } \quad \sin \phi_{x}=\frac{d v}{d x}=v_{x}^{\prime} .
$$

Using Pythagorean trigonometric identity in Eqs (3), one can get:

$$
\begin{aligned}
u_{x}^{\prime}(x, t)=1-\sqrt{1-v_{x}^{\prime}(x, t)^{2}} & \approx \frac{1}{2} v_{x}^{\prime}(x, t)^{2} \\
& \text { and } \quad u_{x}(x, t)=\frac{1}{2} \int_{0}^{x} v_{x}^{\prime}(x, t)^{2} d x .
\end{aligned}
$$

The beam slope has been derived from the second of Eq. (3) as:

$$
\phi_{x}(x, t)=\arcsin v_{x}^{\prime}(x, t) \approx v_{x}^{\prime}(x, t)+\frac{1}{6} v_{x}^{\prime}(x, t)^{3} .
$$

Differentiating the Eq. (5) gave the curvature:

$$
\kappa(x, t)=\phi_{x}^{\prime}(x, t)=\frac{v_{x}^{\prime \prime}(x, t)}{\sqrt{1-v_{x}^{\prime}(x, t)^{2}}} \approx v_{x}^{\prime \prime}(x, t)\left(1+\frac{1}{2} v_{x}^{\prime}(x, t)^{2}\right) .
$$

For the analysed the single degree of freedom model, the deflection of the beam was assumed as:

$$
v_{x}(x, t)=v_{x}(L, t) \psi(x)=v(t) \psi(x)
$$

where $\psi(x)$ represents a single shape mode displacement [23]:

$$
\psi(x)=1-\cos \left(\frac{\pi x}{2 L}\right) .
$$

Substituting Eq. (7) into Eq. (6) the curvature reads:

$$
\kappa(x, t)=v(t) \psi^{\prime \prime}(x)\left(1+\frac{1}{2}\left(v(t) \psi^{\prime}(x)\right)^{2}\right) .
$$


Next it found expression the energies in terms of $v(t)$ :

$$
\begin{aligned}
T= & \frac{1}{2} \rho A \int_{0}^{L}\left[\dot{v}(t)^{2} \psi(x)^{2}+\left(\dot{\chi}(t)-v(t) \dot{v}(t) \int_{0}^{x} \psi^{\prime}(x)^{2} d x\right)^{2}\right] d x \\
& +\frac{1}{2} m_{t}\left[\dot{v}(t)^{2}+\left(\dot{\chi}(t)-v(t) \dot{v}(t) \int_{0}^{L_{t}} \psi^{\prime}(x)^{2} d x\right)^{2}\right] \\
& +\frac{1}{2} I_{0} \dot{v}(t)^{2} \psi^{\prime}\left(L_{t}\right)^{2}\left(1+\frac{1}{2} v(t)^{2} \psi^{\prime}\left(L_{t}\right)^{2}\right)^{2} .
\end{aligned}
$$

and

$$
\begin{aligned}
\Pi= & \frac{1}{2} E I \int_{0}^{L}\left(v(t) \psi^{\prime \prime}(x)+\frac{1}{2} v(t)^{3} \psi^{\prime}(x)^{2} \psi^{\prime \prime}(x)\right)^{2} d x \\
& -\frac{1}{2} \rho A g v(t)^{2} \int_{0}^{L}\left(\int_{0}^{x} \psi^{\prime}(x)^{2} d x\right) d x-\frac{1}{2} m_{t} g v(t)^{2} \int_{0}^{L_{t}} \psi^{\prime}(x)^{2} d x,
\end{aligned}
$$

where the prime denotes differentiation with respect to $x$.

Introducing the displacement function $\psi(x)$ expressed by Eq. (8) and denoting the constants occur in Eq. (10) and Eq. (11) by $N_{1}-N_{8}$, the energies are as follow:

$$
\begin{aligned}
T= & \frac{1}{2} \rho A\left[\dot{v}(t)^{2} N_{1}+\dot{\chi}(t)^{2} L-2 \dot{\chi}(t) v(t) \dot{v}(t) N_{2}+v(t)^{2} \dot{v}(t)^{2} N_{3}\right] \\
& +\frac{1}{2} m_{t}\left[\dot{v}(t)^{2}+\dot{\chi}(t)^{2}-2 \dot{\chi}(t) v(t) \dot{v}(t) N_{4}+v(t)^{2} \dot{v}(t)^{2} N_{4}^{2}\right] \\
& +\frac{1}{2} I_{0} \dot{v}(t)^{2} N_{5}^{2}\left(1+\frac{1}{2} v(t)^{2} N_{5}^{2}\right)^{2},
\end{aligned}
$$

and

$$
\Pi=\frac{1}{2} E I\left(v(t)^{2} N_{6}+v(t)^{4} N_{7}+\frac{1}{4} v(t)^{6} N_{8}\right)-\frac{1}{2} g v(t)^{2}\left(\rho A N_{2}+m_{t} N_{4}\right) .
$$

Above constants $N_{1}-N_{8}$ are:

$$
\begin{aligned}
& N_{1}=\int_{0}^{L} \psi(x)^{2} d x=L\left(\frac{3}{2}-\frac{4}{\pi}\right) \\
& N_{2}=\int_{0}^{L} \int_{0}^{x} \psi^{\prime}(x)^{2} d x d x=\frac{\pi^{2}-4}{16} \\
& N_{3}=\int_{0}^{L}\left(\int_{0}^{x} \psi^{\prime}(x)^{2} d x\right)^{2} d x=\frac{2 \pi^{4}-9 \pi^{2}}{384 L}, \\
& N_{4}=\int_{0}^{L_{t}} \psi^{\prime}(x)^{2} d x=\frac{\pi^{2}}{8 L_{t}}, \\
& N_{5}=\psi^{\prime}\left(L_{t}\right)=\frac{\pi}{2 L_{t}}, \\
& N_{6}=\int_{0}^{L} \psi^{\prime \prime}(x)^{2} d x=\frac{\pi^{4}}{32 L^{3}}, \\
& N_{7}=\int_{0}^{L} \psi^{\prime \prime}(x)^{2} \psi^{\prime}(x)^{2} d x=\frac{\pi^{6}}{512 L^{5}} \\
& N_{8}=\int_{0}^{L} \psi^{\prime \prime}(x)^{2} \psi^{\prime}(x)^{4} d x=\frac{\pi^{8}}{4096 L^{7}} .
\end{aligned}
$$


Using the Lagrange approach $\frac{d}{d t}\left(\frac{\partial \mathbb{L}}{\partial \dot{v}}\right)-\frac{\partial \mathbb{L}}{\partial v}=0$, where $L=T-\Pi$ is the Lagrangian and neglecting in the calculations the third and higher order terms, the Eq. of motion reads as follow:

$$
\begin{gathered}
{\left[m_{t}+\rho A N_{1}+I_{0} N_{5}^{2}+\left(\rho A N_{3}+m_{t} N_{4}^{2}+I_{0} N_{5}^{4}\right) v^{2}\right] \ddot{v}+\left[\rho A N_{3}+m_{t} N_{4}^{2}+I_{0} N_{5}^{4}\right] v \dot{v}^{2}} \\
+\left[E I\left(N_{6}+2 N_{7} v^{2}\right)-(g+\ddot{\chi})\left(m_{t} N_{4}+\rho A N_{2}\right)\right] v=0
\end{gathered}
$$

where $\ddot{\chi}$ determines the acceleration of the beam base according to the type of noise. It has two following forms, by included a parametric noise, then $\ddot{\chi}=\ddot{\chi}_{1}$ (Eq. (16)) and an additive noise, then $\ddot{\chi}=\ddot{\chi}_{2}$ (Eq. (17)), respectively:

$$
\begin{aligned}
& \ddot{\chi}_{1}=A_{e}\left(2 \pi f_{e}\right)^{2} \cos \left(2 \pi f_{e} t+\Gamma(t)\right) \\
& \ddot{\chi}_{2}=A_{e}\left(2 \pi f_{e}\right)^{2} \cos \left(2 \pi f_{e} t\right)+\Gamma(t)
\end{aligned}
$$

where parameters $f_{e}$ and $A_{e}$ are excitation frequency and amplitude, respectively and $\Gamma(t)$ denotes stochastic force with standard deviation $\sigma_{F}$.

Presented in Fig. 1 model was mounted on the base in position which created conditions for different behaviours while system vibrated. The expected behaviours depend on three equilibrium positions of the beam. The first one beam reached at non-buckled state. It occurs by introduced zero values of acceleration and velocity in Eq. (15). Then one can get one trivial solution $v=0$. The others two equilibrium positions beam reached at buckled state. It depends on below inequality:

$$
E I N_{6}<g\left(m_{t} N_{4}+\rho A N_{2}\right)
$$

If Eq. (18) is satisfied, the non zero stable equilibrium solutions are

$$
v_{0}= \pm \sqrt{\frac{g\left(m_{t} N_{4}+\rho A N_{2}\right)-I E N_{6}}{2 E I N_{7}}} .
$$

The natural frequency of the beam $f_{n}$ changes due to the set of the beam equilibrium position. For solution $v=0$, the linearised Eq. of motion for free response about the equilibrium point reads:

$$
\left[m_{t}+\rho A N_{1}+I_{0} N_{5}^{2}\right] \ddot{v}+\left[E I N_{6}-g\left(m_{t} N_{4}+\rho A N_{2}\right)\right] v=0
$$

and after short calculations, from Eq. (20) one can get the natural frequency:

$$
f_{n 1}=\frac{1}{2 \pi} \sqrt{\frac{E I N_{6}-g\left(m_{t} N_{4}+\rho A N_{2}\right)}{m_{t}+\rho A N_{1}+I_{0} N_{5}^{2}}} .
$$

When the beam takes a buckled position $\left(v=v_{0 b}\right)$, the linearised Eq. of motion for free response becomes:

$$
\left[m_{t}+\rho A N_{1}+I_{0} N_{5}^{2}+\left(\rho A N_{3}+m_{t} N_{4}^{2}+I_{0} N_{5}^{4}\right) v_{0 b}^{2}\right] \ddot{\eta}+4 E I N_{7} v_{0 b}^{2} \eta=0
$$


Table 1. Physical and geometric properties of the bimorph cantilever beam.

\begin{tabular}{ll}
\hline Symbol and value & Description \\
\hline$L=0.3 \mathrm{~m}$ & length of the beam \\
$m_{t}=0.02 \mathrm{~kg}$ & the tip mass \\
$m_{b}=0.0096 \mathrm{~kg}$ & the beam mass \\
$\rho=7850 \mathrm{~kg} / \mathrm{m}^{3}$ & density of the beam mass \\
$A=4.064 \mathrm{~mm}^{2}$ & area of transverse beam \\
$E=210 \mathrm{GPa}$ & Young modulus \\
$I=2.18 \times 10^{-14} \mathrm{~m}^{4}$ & geometrical moment of inertia \\
$I_{0}=0.0018 \mathrm{~kg} \mathrm{~m}^{2}$ & the inertia moment of beam $m_{b}$ with tip mass $m_{t}$ \\
$C_{p}=38.9 \mathrm{nF}$ & capacitance of the piezoelectric patches \\
$R=50 \mathrm{k} \Omega$ & load resistance \\
$L_{p}=28 \mathrm{~mm}$ & active length of piezoelectric layers \\
$b_{b}=16 \mathrm{~mm}$ & beam width \\
$h_{b}=0.254 \mathrm{~mm}$ & beam thickness \\
$h_{p}=300 \mu \mathrm{m}$ & piezoelectric layers thickness \\
$e_{31}=0.1105 \mathrm{C} / \mathrm{m}^{2}$ & piezoelectric constant \\
$D_{1}=48.08 \times 10^{-6} \mathrm{~N} / \mathrm{V}$ & electromechanical coupling constant \\
$D_{2}=0.07 \times 10^{-6} \mathrm{~N} / \mathrm{V}$ & electromechanical coupling constant \\
\hline
\end{tabular}
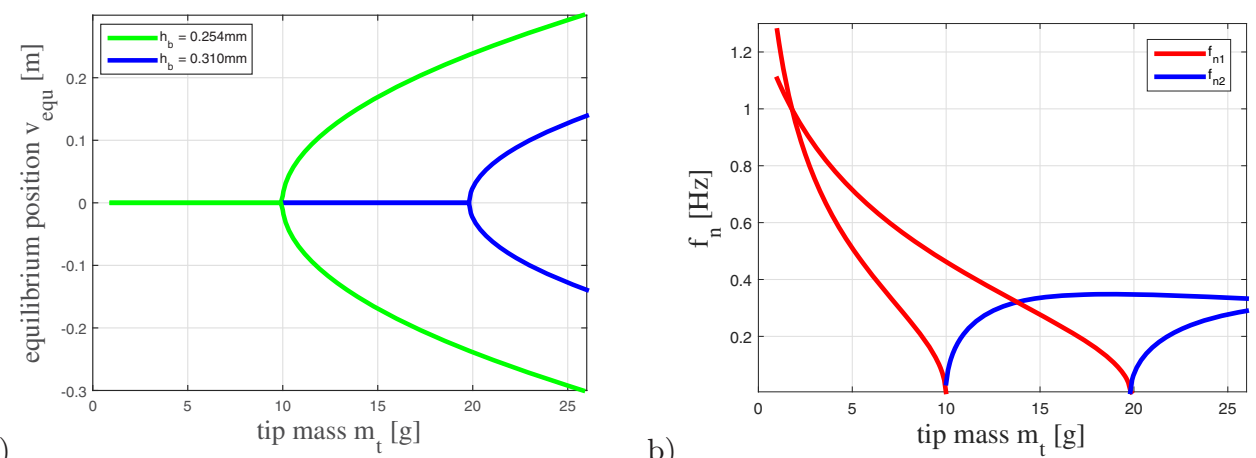

a)

Fig. 2. The pitchfork bifurcation diagram of equilibrium positions $v_{\text {equ }}$ (a) and the natural frequencies $f_{n}$ against tip mass $m_{t}$ depended on beam thickness $h_{b}$.

where $v=v_{0 b}+\eta$, and for both the left and the right buckled equilibrium positions of the beam, the natural frequency is:

$$
f_{n 2}=\frac{1}{2 \pi} \sqrt{\frac{4 E I N_{7} v_{o b}^{2}}{m_{t}+\rho A N_{1}+I_{0} N_{5}^{2}+\left(\rho A N_{3}+m_{t} N_{4}^{2}+I_{0} N_{5}^{4}\right) v_{0 b}^{2}}} .
$$

The significant influence on the natural frequencies as well the equilibrium positions has the tip mass $m_{t}$. For the adopted parameters of the beam, listed in the Table 1, the behaviour changed from single solution via bifurcation point the new solutions appeared. It is observed at tip mass $m_{t} \approx 10 \mathrm{~g}$ (or $m_{t} \approx 20 \mathrm{~g}$ when the beam thickness is $22 \%$ larger). Figure $2 \mathrm{a}$ presents the points, where system was undergoing from equilibrium position in $v_{0}=0$ into buckled equilibrium states $v_{0 b} \neq 0$. The natural frequencies of the system one can observe in Fig. 2b for non-buckled (red lines) and buckled beam positions (blue lines). The interesting both points at $f_{n} \approx 0$ for different 
$h_{b}$ dimension, coincides with the bifurcation of the equilibrium positions. In further analysis the $h_{b}$ dimension was fixed to get the bifurcation point at tip mass $m_{t} \approx 10 \mathrm{~g}$.

The coupled piezoelectric model leans on the derivation of the electro mechanical model provided by authors [13]. The electrical Eq. is combined with the mechanical Eq. of motion 15 and reads:

$$
\begin{aligned}
& {\left[m_{t}+\rho A N_{1}+I_{0} N_{5}^{2}+\left(\rho A N_{3}+m_{t} N_{4}^{2}+I_{0} N_{5}^{4}\right) v^{2}\right] \ddot{v}+\left[\rho A N_{3}+m_{t} N_{4}^{2}+I_{0} N_{5}^{4}\right] v \dot{v}^{2}} \\
& \quad+\left[E I\left(N_{6}+2 N_{7} v^{2}\right)-(g+\ddot{\chi})\left(m_{t} N_{4}+\rho A N_{2}\right)\right] v-\left(D_{1}+D_{2} v^{2}\right) U=0 \\
& C_{p} \dot{U}+\frac{U}{R}+\left(D_{1}+D_{2} v^{2}\right) \dot{v}=0
\end{aligned}
$$

where $R$ is the load resistance, $C_{p}$ is the capacitance of the piezoelectric patches and $U$ is the voltage across the load resistor connected to the piezoelectric patches. The beam is equipped with piezoelectric patches and connected in parallel. For coupling the mechanical and the electrical subsystems, the electromechanical constants $D_{1}$ and $D_{2}$ have been introduced, in case of linear coupling written as:

$$
D_{1}=e_{31} b_{p}\left(h_{p}+h_{b}\right) \int_{0}^{L_{p}} \psi^{\prime \prime}(x) \mathrm{d} x=e_{31} b_{p}\left(h_{p}+h_{b}\right) \psi^{\prime}\left(L_{p}\right)
$$

and nonlinear one written as:

$$
D_{2}=\frac{3 e_{31} b_{p}\left(h_{p}+h_{b}\right)}{2 \times 10^{6}} \int_{0}^{L_{p}} \psi^{\prime \prime}(x) \psi^{\prime}(x)^{2} \mathrm{~d} x=\frac{e_{31} b_{p}\left(h_{p}+h_{b}\right)}{2 \times 10^{6}} \psi^{\prime}\left(L_{p}\right)^{3} .
$$

Dimensions $h_{p}$ and $h_{b}$ denote the piezoelectric and beam thickness, respectively, $b_{p}$ is the piezoelectric layer width, $L_{p}$ denotes an active length of piezoelectric layer, and $e_{31}$ is the piezoelectric constant. The piezoelectric patches produce the charge given by $D_{1} v+D_{2} v^{3}$.

Finally, using the approximation for curvature $\kappa(x, t)$ in Eq. (6), and the displacement model in Eq. (7), the moving and extracting piezoelectric patches carry out the work $W$ :

$$
W \approx\left(D_{1} v+\frac{1}{3} D_{2} v^{3}\right) U
$$

From simulated voltage $U$, the averaged power scavenged between times $t_{1}$ and $t_{2}$ is obtained from:

$$
P_{\text {ave }}=\frac{1}{t_{2}-t_{1}} \int_{t_{1}}^{t_{2}} \frac{U(t)^{2}}{R} \mathrm{~d} t
$$

where $t_{2}-t_{1}=\Delta t$ is a number of each simulation time periods $T$.

\section{The numerical simulation results}

The simulations of the system presented in Fig. 1, based on Eq. (24) and system parameters (Table 1) have been performed using Matlab environments. The random force, included in the excitation functions (Eq. (16)) and (Eq. (17)) has been modelled by means of Runge Kutta Murayama algorithm and used white noise of Gaussian 
a)

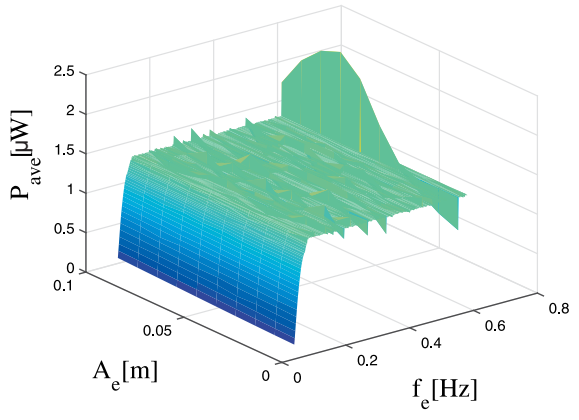

b)

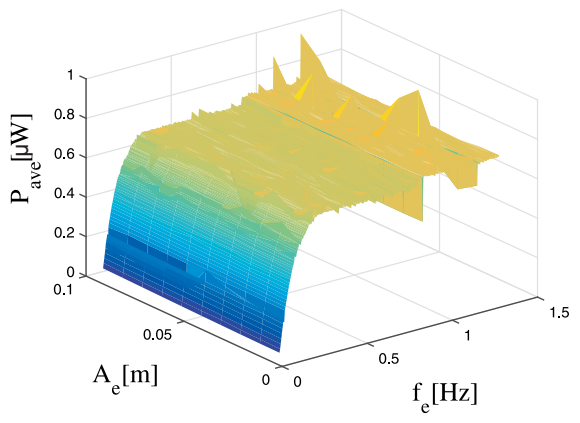

Fig. 3. The averaged power $P_{\text {ave }}$ of the system against excitation amplitude $A_{e}$ and frequency $f_{e}$ at no noise component included in the excitation $\sigma_{F}=0$ for tip masses $m_{t}=10.5 \mathrm{~g}$ (a) and $m_{t}=20 \mathrm{~g}(\mathrm{~b})$, respectively.

distribution. The simulation time was assumed as 700 and 400 times of period $T=f_{e}^{-1}$, in cases of $D_{2}=0$ and $D_{2} \neq 0$, respectively. While the time step was $d t=0.01 T$. The shorter time simulation in case of $D_{2} \neq 0$ was adopted due to escape phenomenon. The initial time intervals of each simulation were neglected as transient time and it was adopted $75 \%$ of whole time simulation. The initial conditions in simulations were $\left[v_{0}=v_{e}, \dot{v}_{0}=0, U_{0}=0\right]$, where $v_{e}$ denotes the equilibrium position and for Euler buckling mass $m_{e b}=\left(E I \pi^{2}\right) /\left(4 g L^{2}\right)$ is:

$$
v_{e}=\frac{2 L}{\pi} \sqrt{2 m_{t} / m_{e b}-2}
$$

In the simulation process for the consecutive loops, the continue method to the initial condition was applied, to reflect the real position of the tip mass. The main results of this work contents of two cases simulations, by assuming linear coupled electrical subsystem to the mechanical one, and nonlinear by introducing the second constant $D_{2}$ as reported in Eq. (24). The linear model case is presented in Figs. 3, 6 and 9 and the nonlinear case is reported in Figs. 11, 12 and 13. These figures represent the maps of averaged power $P_{\text {ave }}$ against the two input parameters, excitation amplitude $A_{e}$ and excitation frequency $f_{e}$. The results present the influence of the different form of excitation forces for the sake of the tip mass $m_{t}$ weight. For review the system behaviours, two tip masses have been adopted for simulation. The first value $m_{t} \approx 10 \mathrm{~g}$ to received the jumps between potential wells and the second $m_{t}=20 \mathrm{~g}$ to got the buckled position.

Figure $3 \mathrm{a}$ and $3 \mathrm{~b}$ present the behaviour of the system subjected to harmonic excitation and no noise disturbance. It is easy to notice, the averaged power $P_{\text {ave }}$ output reached the higher values in case of implemented tip mass (Fig. 3a), where the beam vibrated around the bifurcation point, between zero and buckled equilibrium positions. In Fig. 4 was shown the behaviour of the system at tip mass $m_{t} \approx 10 \mathrm{~g}$, excitation amplitude $A_{e}=0.07 \mathrm{~m}$ and excitation frequency $f_{e}=0.61 \mathrm{~Hz}$. Such input parameters reflected the peak of power $P_{\text {ave }}$ visible in Fig. 3a. The time series and phase portrait revealed the beam rocking around the double potential wells. Fig. 4c confirm the movement of the tip mass between the potential wells by increasing jumps number in whole time simulation. Such behaviour caused the high stress of the piezoelectric patches and finally raised efficiency of energy harvesting. In case of the larger tip mass at $m_{t}=20 \mathrm{~g}$, the jumps between potential wells did not occur. It led to single well dynamics. In Figs. $5 \mathrm{a}-\mathrm{c}$ one can see the motion, which took place on the right side buckled position by $A_{e}=0.05 \mathrm{~m}$ and $f_{e}=0.35 \mathrm{~Hz}$. The time series 
a)

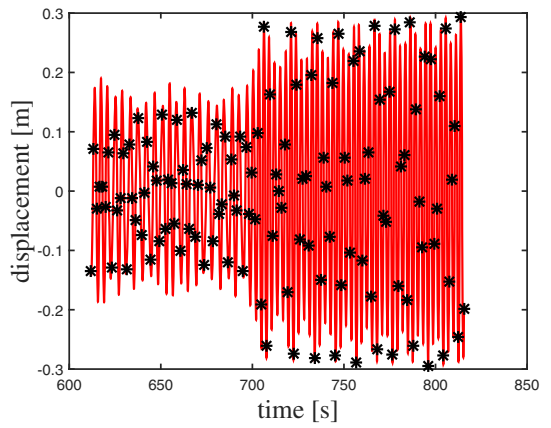

b)

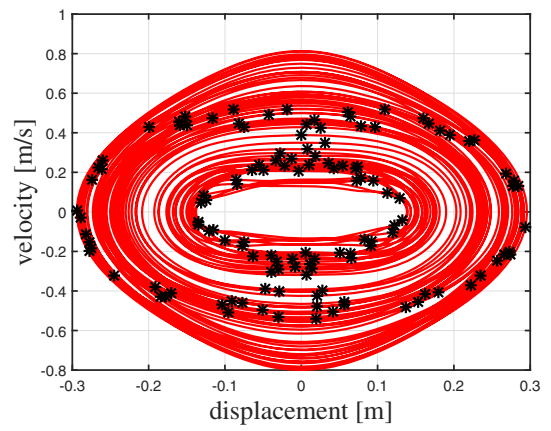

c)

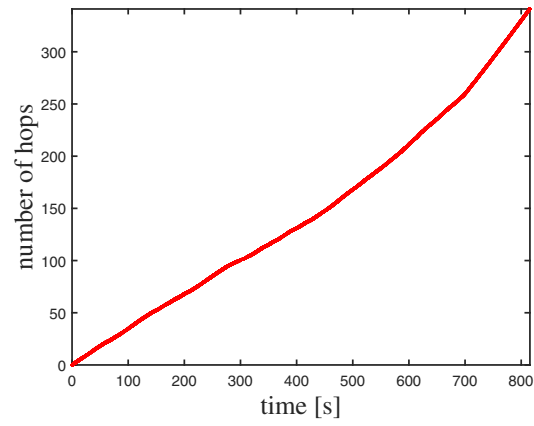

Fig. 4. The time series of displacement (a), phase portrait of the system with tip mass $m_{t}=10.5 \mathrm{~g}$ (b) and number of jumps between the potential well (c) against simulation time at no noise component included in the excitation.

present the beam motion like a rumble effect in a single potential well. In the next Figs. $6 \mathrm{a}$ and $6 \mathrm{~b}$, the influence of noise component is considered. The tip mass was adopted $m_{t}=20 \mathrm{~g}$ to kept motion in a single potential well, and the system was excited harmonically according to functions in Eq. (16) and Eq. (17), where the two ways of disturbance were included. Following the results, presented in Fig. 6, the influence of noise at level $\sigma_{F}=0.1$ is more visible in case of an additive form in excitation formula than the noise added as a argument of cosine function. In case of the excitation according to Eq. (16), the output averaged power $P_{\text {ave }}$ created a smooth map. Here one can expect a constant level of output power. The disturbance of input noise signal has negligible effect, comparing to the result presented in Fig. 3b. The time series and phase portrait presented in Figs. 7a and $7 \mathrm{~b}$ plotted at parameters $A_{e}=0.01 \mathrm{~m}$, and $f_{e}=1.4 \mathrm{~Hz}$ show, there were also no jumps between potential wells, and the system behaved similar to the case of harmonic excitation with no noise (Fig. 5). The second way of the system excitation according to Eq. (17) appeared a different solution. Introducing the noise to the excitation as a separate signal, it provided more visible peaks on the averaged power $P_{\text {ave }}$ map (Fig. 6b). Moreover, the trajectory of the tip mass moved at the beginning in a single well (Fig. 8c), finally there was observed the intermittent displacement which followed to the motion with jumps between potential wells what is shown in Figs. 8. The chosen parameters used in Fig. 8 are $A_{e}=0.01 \mathrm{~m}$, and $f_{e}=1.4 \mathrm{~Hz}$. Analysing the influence of an additive excitation noise, one can conclude, it provides conditions for raise the efficiency level of energy harvesting.

Finally, in Figs. 9a and 9b the results of the implemented higher noise level $\sigma_{F}=$ 0.5 in the excitation formula were presented. The system excited with increased noise component effected an appearance of extremely peaks of averaged power $P_{\text {ave }}$. In this 

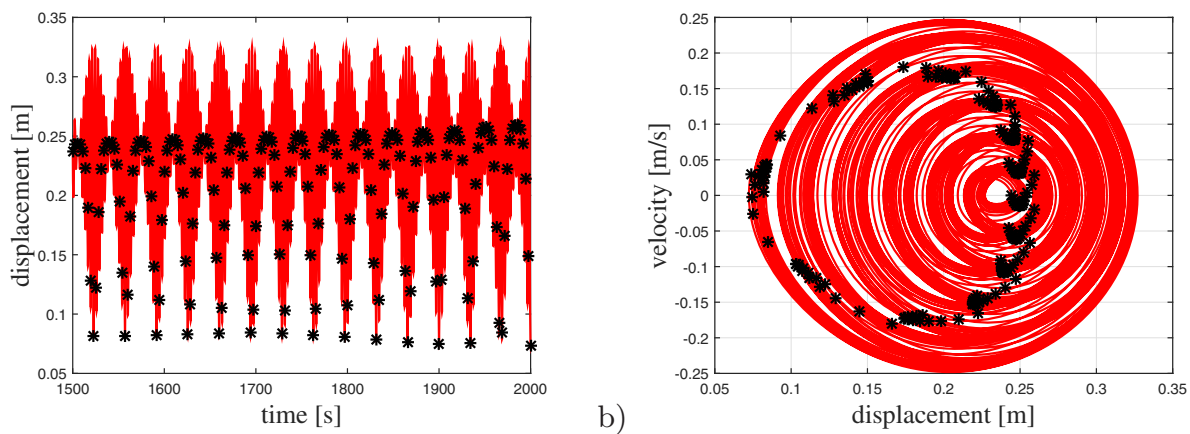

a)

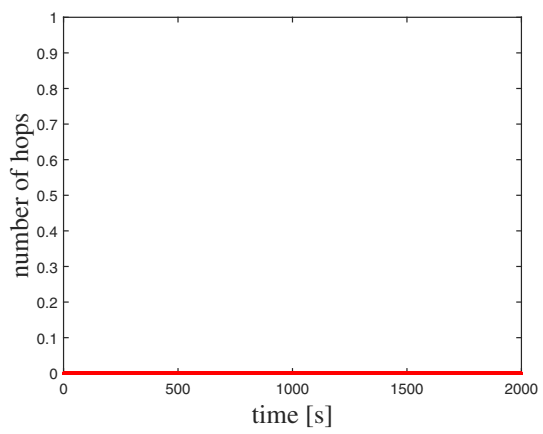

Fig. 5. The time series of displacement (a), phase portrait of the system with tip mass $m_{t}=20 \mathrm{~g}$ (b) and number of jumps between the potential well (c) against simulation time at no noise component included in the excitation.

a)

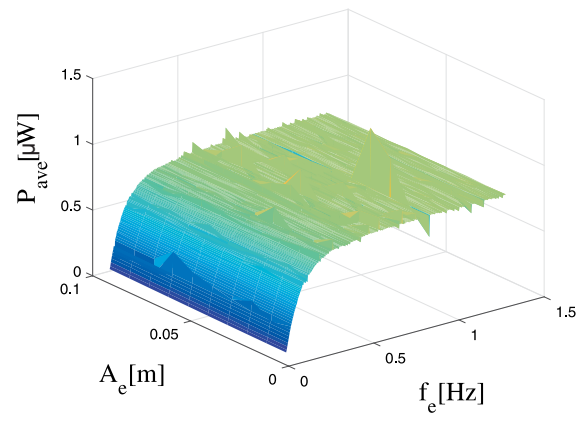

b)

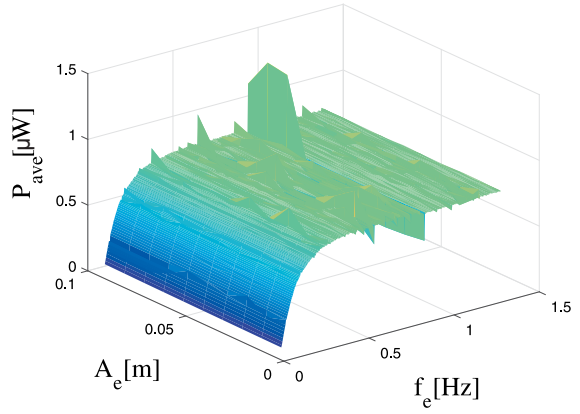

Fig. 6. The averaged power $P_{a v e}$ of the system against excitation amplitude $A_{e}$ and frequency $f_{e}$ for tip mass $m_{t}=20 \mathrm{~g}$ at noise component $\sigma_{F}=0.1$ included in the excitation according to Eq. (16) (a), and according to Eq. (17) (b), respectively.

case, the excitation amplitudes had no significant influence on the solutions, but the excitation frequency had.

During simulation, the increased input frequency caused maxima of $P_{\text {ave }}$ visible both in Figs. 9a and 9b. The beam vibrated over double wells, what was confirmed in Fig. 10 at parameters $A_{e}=0.01 \mathrm{~m}$ and $f_{e}=0.5 \mathrm{~Hz}$. Moreover, either increased the noise level $\sigma_{F}$ or used disturbances as an additive case of noise only, the verification in chosen points on maps, revealed escapes from the potential wells. The time series of displacement went beyond the beam length relative to the support point 
a)

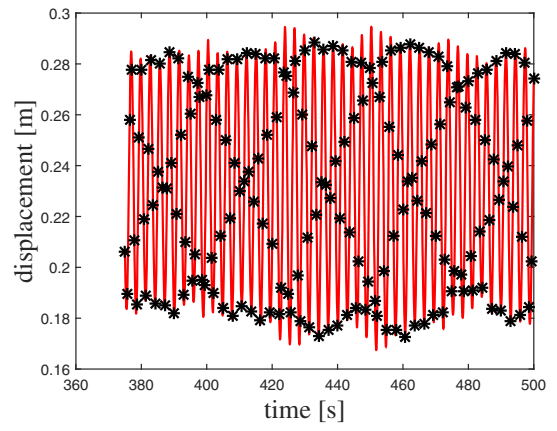

b)

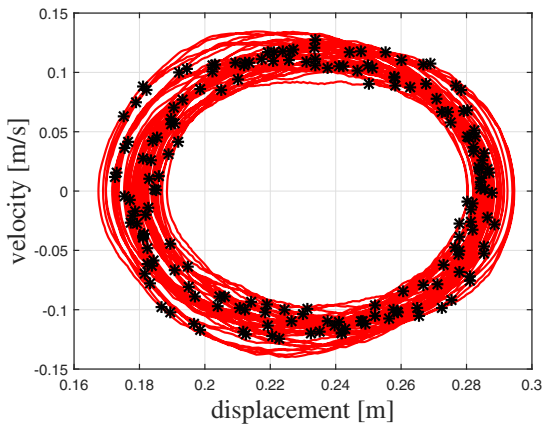

Fig. 7. The time series of displacement (a) and phase portrait of the system with tip mass $m_{t}=20 \mathrm{~g}$ (b) at noise component $\sigma_{F}=0.1$ included to the cosine function in the excitation according to Eq. (16).

a)

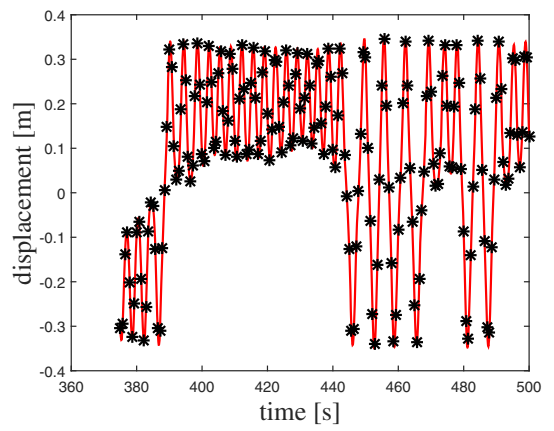

b)

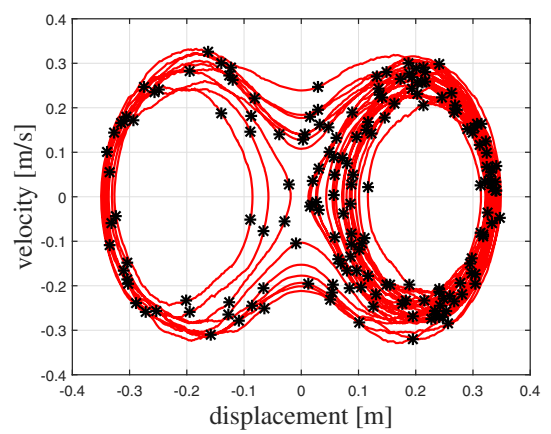

c)

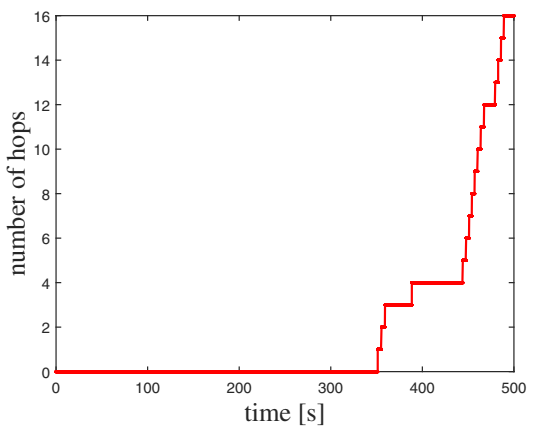

Fig. 8. The time series of displacement (a), phase portrait of the system with tip mass $m_{t}=20 \mathrm{~g}$ (b) and number of jumps between the potential well (c) against simulation time at noise component $\sigma_{F}=0.1$ included as an additive excitation according to Eq. (17).

(see Fig. 8). That phenomenon appeared mainly due to high level of noise adopted to the excitation. On the other hand such behaviour creates well conditions for energy harvesting because the system works away from resonance region.

The next simulations, presented in Figs. 11, 12 and 13 revealed the nonlinear modelling of piezoelectric subsystem by means of the electromechanical constants $D_{2} \neq 0$. Figures 11 report a gradually increasing $P_{\text {ave }}$ in both cases of tip masses, while the same simulation but in liniar moddeling of piezoelectric (Fig. 3), the power had rather constant value. Figures $12 \mathrm{a}$ and b represent the output power, also gradually increasing $P_{\text {ave }}$ during frequency excitation was growing, while at $D_{2}=0$ increasing of the $P_{\text {ave }}$ occurred faster (see Fig. 6). There was also noticed more peaks in case of the 
a)

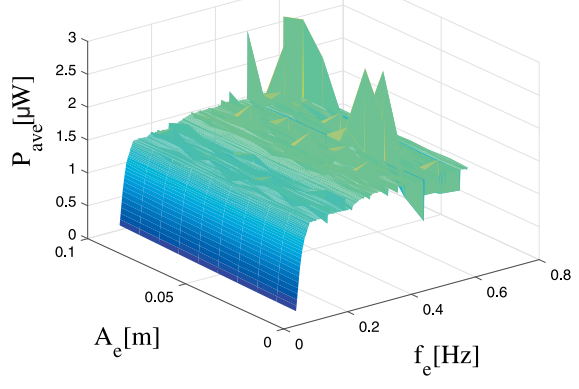

b)

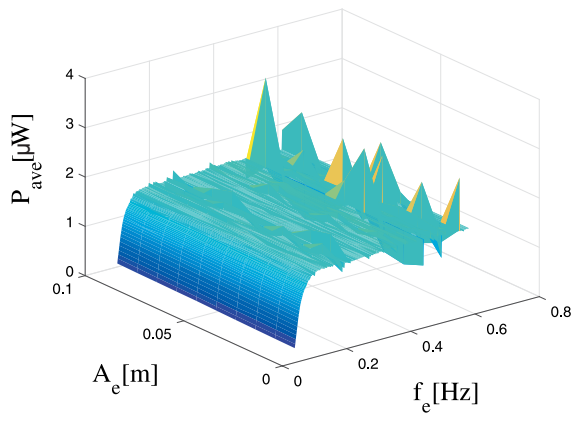

Fig. 9. The averaged power $P_{a v e}$ of the system against excitation amplitude $A_{e}$ and frequency $f_{e}$ for tip mass $m_{t}=10.5 \mathrm{~g}$ at noise component $\sigma_{F}=0.5$ included in the excitation according to Eq. (16) (a), and according to Eq. (17) (b), respectively.

a)

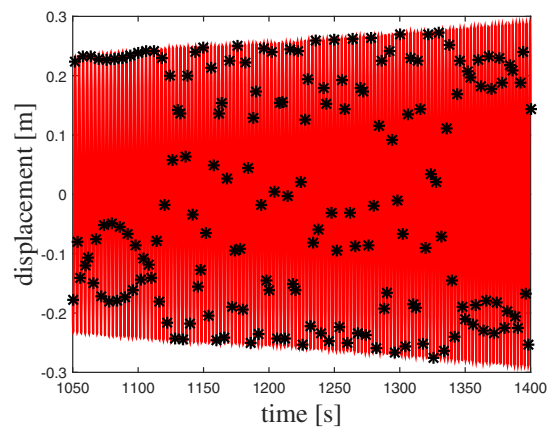

b)

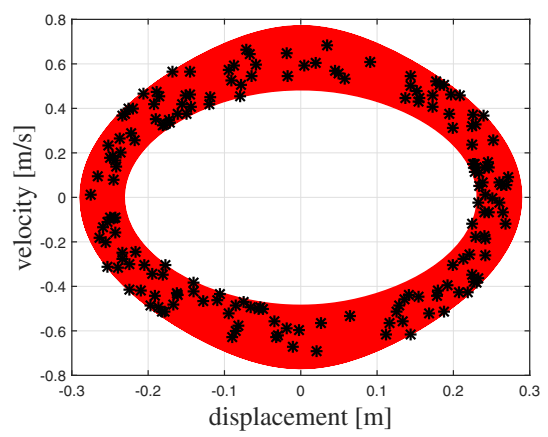

c)

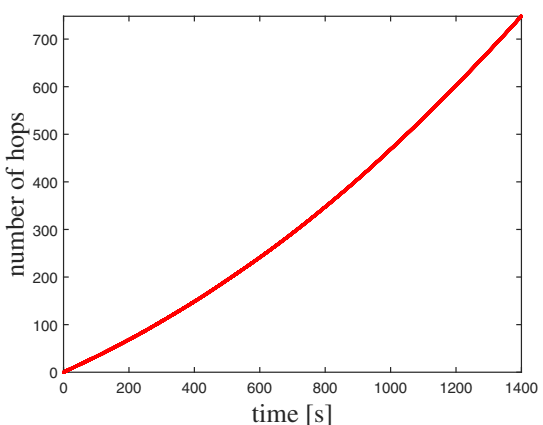

Fig. 10. The time series of displacement (a), phase portrait of the system with tip mass $m_{t}=10.5 \mathrm{~g}$ (b) and number of jumps between the potential well (c) against simulation time at noise component $\sigma_{F}=0.5$ included to the cosine function in the excitation according to Eq. (16).

harmonic excitation, disturbed by additive noise component (Fig. 12b). It could be due to a greater system sensitivity to the modelling approach including both coupling constants, $D_{1}$ and $D_{2}$. Unfortunately, there are no significant differences between maps presented in Fig. 9 against the maps in Fig. 13 where the linear and nonlinear piezoelectric modelling cases have been reported, respectively. But it is easy to notice, that in case of system worked at bifurcation point, the energy harvesting efficiency 
a)

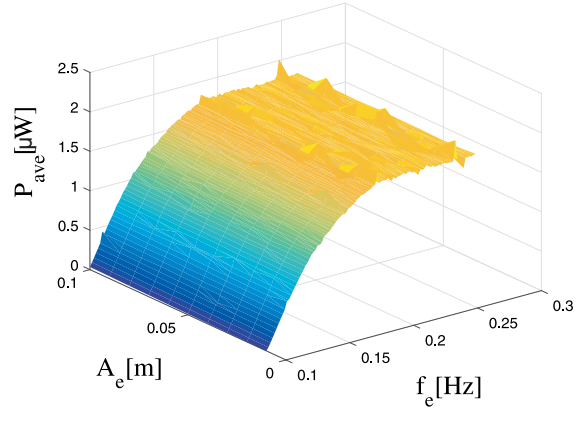

b)

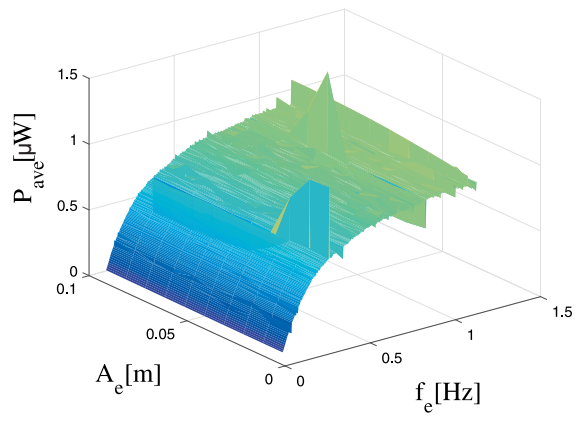

Fig. 11. The averaged power $P_{\text {ave }}$ of the system against excitation amplitude $A_{e}$ and frequency $f_{e}$ at no noise component included in the excitation $\sigma_{F}=0$ for tip masses $m_{t}=$ $10.5 \mathrm{~g}(\mathrm{a})$ and $m_{t}=20 \mathrm{~g}(\mathrm{~b})$, respectively.

a)

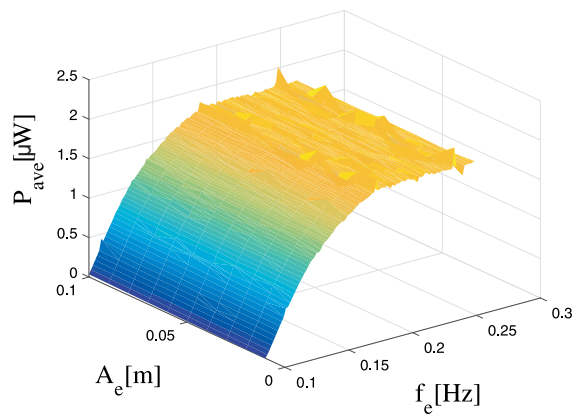

b)

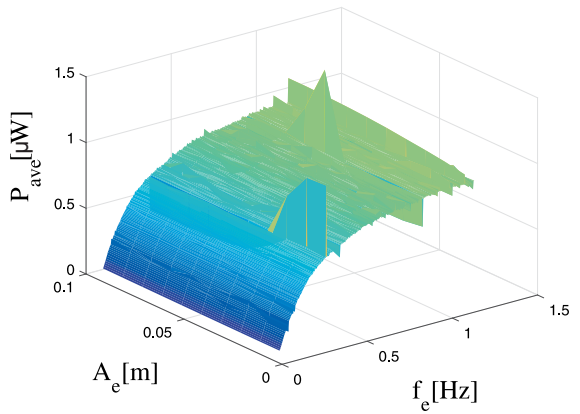

Fig. 12. The averaged power $P_{a v e}$ of the system against excitation amplitude $A_{e}$ and frequency $f_{e}$ for tip mass $m_{t}=20 \mathrm{~g}$ at noise component $\sigma_{F}=0.1$ included in the excitation according to Eq. (16) (a), and according to Eq. (17) (b), respectively.

a)

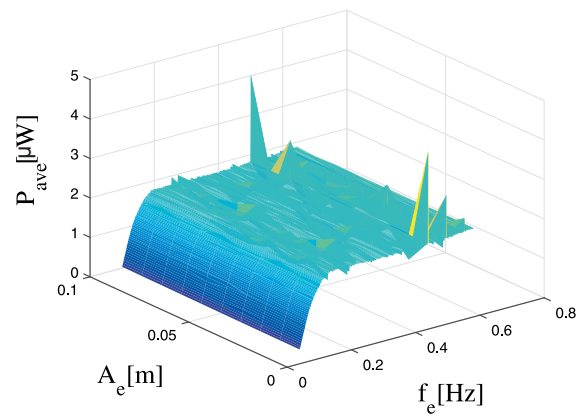

b)

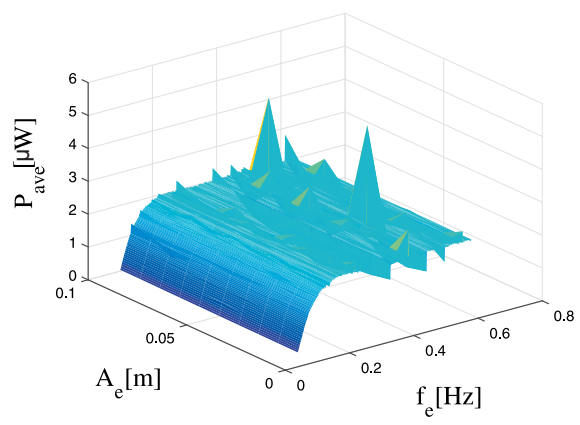

Fig. 13. The averaged power $P_{a v e}$ of the system against excitation amplitude $A_{e}$ and frequency $f_{e}$ for tip mass $m_{t}=10.5 \mathrm{~g}$ at noise component $\sigma_{F}=0.1$ included in the excitation according to Eq. (16) (a), and according to Eq. (17) at $\sigma_{F}=0.5$ (b), respectively.

came in the similar higher level for both, linear and nonlinear model, comparing to other maps as in Fig. 12a and b. The last Figs. 14a-d present comparison the output power level from both, linear and nonlinear piezoelectric model. It clearly shows, the averaged power got a higher values when coupling constant $D_{2} \neq 0$ (Fig. 14c) in relation to the linear case $D_{2}=0$ (Fig. 14a). Such behaviour was especially observed while the system vibrated around the bifurcation point $\left(m_{t} \approx 10 \mathrm{~g}\right)$, and also around 
a)
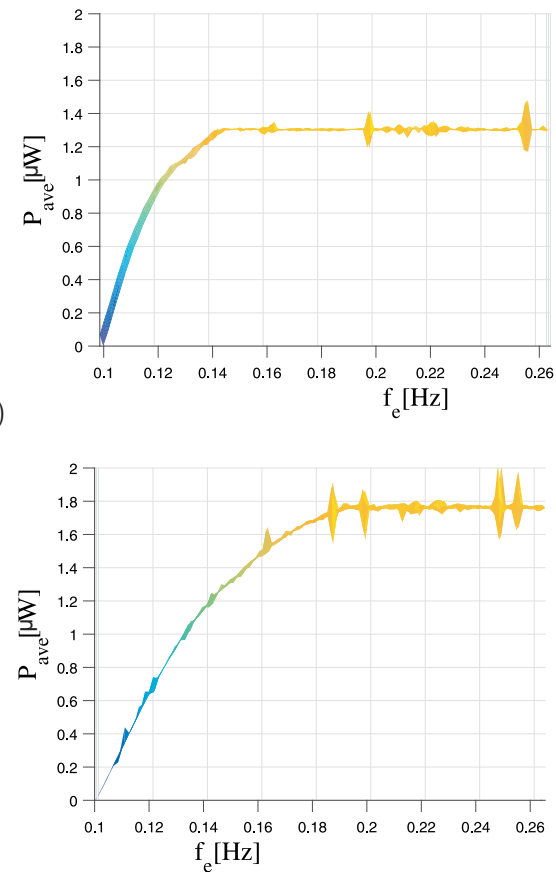

b)
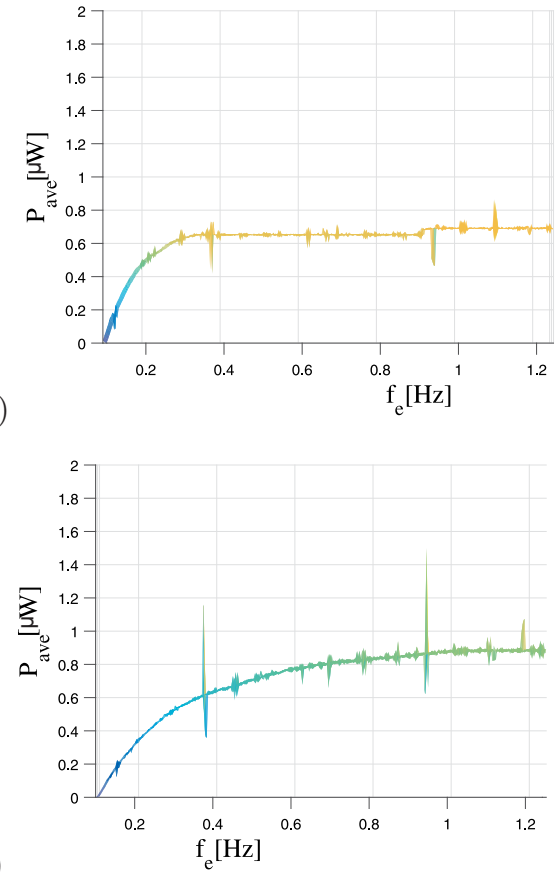

Fig. 14. The averaged power $P_{a v e}$ of the system against the frequency $f_{e}$ at no noise component $\sigma_{F}=0$ for tip mass $m_{t}=10.5 \mathrm{~g}$ (a) and (c) and for tip mass $m_{t}=20 \mathrm{~g}$ (b) and (d). Plots (a) and (c) correspond to the linear case of piezo, but plots (b) and (d) show the nonlinear case of piezo, respectively.

buckled equilibrium position when $m_{t}=20 \mathrm{~g}$. Figures $14 \mathrm{~b}$ and $14 \mathrm{~d}$ correspond to the buckled vibrations. In this case the power revealed less differences between linear and nonlinear model. Comparing the power in Fig. 14a to the power in 14b as well as the power in Fig. 14c to the plot in Fig. 14d, one can notice a significant difference of the output power level. This analysis concludes the energy harvesting efficiency increases at bifurcation point sets of parameters.

\section{The conclusions}

The excitation combined effect was examined in the harmonic and stochastic piezoelectric system of energy harvesting for both, the linear and nonlinear modelling of piezoelectric element. The effect of the averaged power $P_{\text {ave }}$ increasing of particular solutions were demonstrated and discussed. It appeared that the different approach of excitation way of the beam system has effects on transition from single to double wells vibrations. The excitation by harmonic function with additional stochastic component affected the efficiency of energy harvesting by increasing the output power, while the noise signal included to the harmonic function had a weak influence on the solution in compare with the no noisy harmonic excitation signal. On the other hand introducing the linear and nonlinear cases of piezoelectric subsystem and comparing results, the efficiency significantly increased while the piezoelectric had nonlinear characteristic. Analysing the energy harvesting effect, one can notice the noise as an additive component in harmonic excitation increases the system efficiency but simultaneously make the system intermittent by disadvantageous escaping from the potential wells. Especially when electric subsystem was nonlinear, the escaping occurred 
more frequently, and the numerical analysis lost the dynamics description. It requires the experiments tests to observe the system behaviours in wide range of parameters sets.

This work has been funded by the National Science Centre under the grant Agreement No. DEC-2012/05/B/ST8/00080.

\section{References}

1. M. Borowiec, Proceedings of the $16^{\text {th }}$ International Conference on Mechatronics Mechatronika 2014, 194 (2014)

2. C.B. Williams, R.B. Yates, Sens. Actuators A 52, 8 (1996)

3. S. Roundy, P. Wright, J. Rabaey, Kluwer Academics Boston M.A. (2003)

4. S.R. Anton, A. Ertruk, D.J. Inmann, Smart Mater. Struct. 19, 115021 (2010)

5. H.S. Kim, J.-H. Kim, J. Kim, Inter. J. Precision Engin. Manufacturing 12, 1129 (2011)

6. P.D. Mitcheson, E.M. Yeatman, G.K. Rao, A.S. Holmes, T.C. Green, Proc. IEEE 96, 1457 (2008)

7. L. Tang, Y. Yang, C.K. Soh, J. Intell. Mater. Syst. Struct. 21, 1867 (2010)

8. A. Erturk, D. Inman, Piezoelectric Energy Harvesting, (John Wiley \& Sons Ltd., Chichester, UK, 2011)

9. S. Priya, J. Electroceram. 19, 167 (2007)

10. A. Erturk, J. Hoffmann, D.J. Inman, Applied Phys. Lett. 94, 254102 (2009)

11. E.S. Leland, P.K. Wright, Smart Mater. Struct. 15, 1413 (2006)

12. G. Litak, M.I. Friswell, S. Adhikari, Applied Phys. Lett. 96, 214103 (2010)

13. M.I. Friswell, S.F. Ali, S. Adhikari, A.W. Lees, O. Bilgen, G. Litak, J. Int. Mat. Syst. Struc. 23, 1505 (2013)

14. M. Borowiec, G. Litak, M.I. Friswell, S.F. Ali, S. Adhikari, A.W. Lees, O. Bilgen, Int. J. Struct. Stability Dyn. 13, 1340006 (2013)

15. E. Halvorsen, J. Micromech. Syst. 17, 1061 (2008)

16. L.-C.J. Blystad, E. Halvorsen, Smart Mater. Struct. 20, 025011 (2011)

17. M. Ferrari, M. Bau, M. Guizzetti, V. Ferrari, Sensor Actuators A-Phys. 172, 287 (2011)

18. G. Litak, M. Borowiec, M.I. Friswell, S. Adhikari, J. Theor. Appl. Mech. 49, 757 (2011)

19. N.A. Khovanova, I.A. Khovanov, App. Phys. Lett. 99, 144101 (2011)

20. M.F. Daqaq, J. Sound Vibr. 330, 2554 (2011)

21. W. Martens, U. von Wagner, G. Litak, Eur. Phys. J. Special Topics 222, 1665 (2013)

22. P. Kumar, S. Narayanan, S. Adhikari, M.I. Friswell, J. Sound Vibr. 333, 2040 (2014)

23. E. Esmailzadeh, G. Nakhaie-Jazar, Int. J. Nonlinear Mech. 33, 567 (1998) 